\title{
Relapsing Cutaneous Multiple Myeloma Responding to Immunochemotherapy: A Rare Case Report
}

\author{
Mujtaba Mohamed ${ }^{\mathrm{a}, \mathrm{b}}$, Alsadiq Alhillan ${ }^{\mathrm{a}}$, Varsha Gupta ${ }^{\mathrm{a}}$, Kenneth Nahumª \\ Arman Mushtaq ${ }^{\mathrm{a}}$, Min Zhenga, Mohammad A. Hossain ${ }^{\mathrm{a}}$
}

\begin{abstract}
Multiple myeloma (MM) is characterized by the neoplastic proliferation of plasma cells. The diagnosis of this disease is often suspected through a constellation of clinical signs and symptoms of hypercalcemia, renal failure, anemia, and M-spike proteins. It is the second most common hematological malignancy after non-Hodgkin lymphomas. However, cutaneous MM is an extremely rare entity, and it is associated with poor prognosis. It presents as diffuse erythematous rash or violaceous nodules on the skin. Most common sites of involvement are chest, lower extremities and back. It can be triggered by a local extension of the tumor which is the most common way, surgical procedures and hematogenous spread. An 82-year-old African American male was diagnosed with MM since 2008. He underwent autologous peripheral stem cell transplantation (ASCT) twice in 2010 and 2014; and he had a history of multiple chemotherapy regimens in the past. He had violaceus chest nodules, and the biopsy confirmed the diagnosis of cutaneous MM in 2013. The patient was treated with pomalidomide, panobinostat and dexamethasone with a complete response (CR) to treatment. One year later, the patient developed new skin nodules. Repeat biopsy confirmed the diagnosis of MM again. Patient was treated with daratumumab and had CR to treatment without any new M-spike. Cutaneous lesion is an exceedingly rare presentation of MM. It either present as reddish rash or violaceous nodules involving chest, lower extremities and back. It has a poor prognosis and can be rapidly fatal. Our case is unique because our patient responded to the newer chemotherapy, and lesions resolved despite poor prognosis of this condition.
\end{abstract}

Keywords: Multiple myeloma; Extramedullary plasmacytoma; $\mathrm{Cu}$ taneous myeloma

\section{Introduction}

Plasma cell proliferative disorders include group of terminally

Manuscript submitted October 4, 2019, accepted October 11, 2019

${ }^{a}$ Department of Medicine, Jersey Shore University Medical Center, Hackensack Meridian Health, Neptune, NJ 07753, USA

${ }^{\mathrm{b}}$ Corresponding Author: Mujtaba Mohamed, Internal Medicine Residency Program, Jersey Shore University Medical Center, Hackensack Meridian Health, Neptune, NJ 07753, USA.

Email: Mujtaba.mohamed@hackensackmeridian.org

doi: https://doi.org/10.14740/jmc3382 differentiated B lymphoid neoplasms characterized by expansion of clonal cells, secreting monoclonal immunoglobulins (Igs) or its fragments in blood or urine [1]. Clinical presentation varies from completely asymptomatic to florid malignancy. Plasma cell proliferative disorders can cause rare and variable dermatologic manifestations. In our case report we focused on a very rare entity, extramedullary plasmacytoma involving skin. Extramedullary plasmacytoma spread through local extension, hematogenous spread and surgical procedures. It usually involves chest, lower extremities and back [2]. Extramedullary plasmacytoma is associated with poor prognosis. Thalidomide efficacy is not well studied due to the rarity of this condition [3]. However, in our patient, it resolved after he received pomalidomide, panobinostat and Decadron on the first time and daratumumab for relapse on the second time.

\section{Case Report}

An 82-year-old African American man presented with anemia and thrombocytopenia in 2009. Skeletal survey showed multiple lytic bone lesions in the rib, scapula, and iliac bone. Bone marrow (BM) trephine biopsy showed $98-100 \%$ cellularity with markedly increased number of plasma cells, essentially replacing the entire marrow (more than $90 \%$ of cellularity). These plasma cells showed cluster of differentiation (CD)138 expression and kappa light chain restriction by immunohistochemistry (IHC). BM flow cytometry demonstrated monoclonal cIgG kappa ${ }^{+}, \mathrm{CD} 38$ bright $^{+}, \mathrm{CD}^{4} 5^{-}$plasma cells. Cytogenetic analysis showed a complex abnormal karyotype, including chromosomal rearrangements involving chromosomes 1, 3, 6 and 9, and loss of chromosomes 13 and 15 . Fluorescence in situ hybridization (FISH) analysis showed monosomy 13 and loss of chromosome 15 . He was diagnosed with IgG kappa myeloma. He underwent five cycles of Revlimid and low-dose dexamethasone, and then underwent one cycle of cyclophosphamide, dexamethasone and etoposide (CDE) debulking chemotherapy. The patient had a clinical and laboratory complete response (CR), so he proceeded with autologous stem cell transplantation (ASCT). In June 2010, he underwent an ASCT and achieved a complete biochemical remission. Skeletal survey showed only degenerative changes. No lytic lesions were identified. BM biopsy showed scattered polyclonal plasma cells based on the kappa and lambda light chain stains. No abnormal plasma cell population was detected by flow cytometry. Cytogenetic analysis showed a normal 


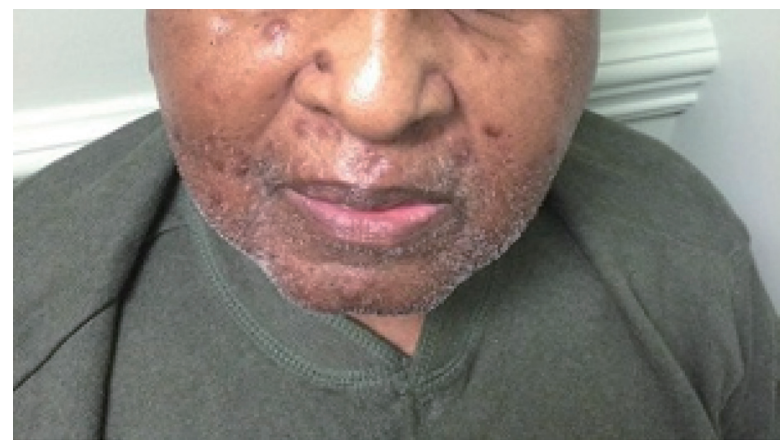

Figure 1. Cutaneous manifestation of multiple myeloma. Patient with relapsed multiple myeloma presenting as nodular erythematous skin eruption.

\section{karyotype.}

In August 2013, he developed a recurrence of MM. Serum IgG was elevated at $4,643 \mathrm{mg} / \mathrm{dL}$. BM biopsy showed $80-90 \%$ plasma cells. Cytogenetic analysis again showed a complex abnormal karyotype, with gain of chromosomes 1 and 3, loss of chromosomes $13,14,15,18,19,20$ and 22, and additional material on chromosomes $1 \mathrm{p}, 3 \mathrm{q}, 8 \mathrm{p}, 11 \mathrm{p}, 12 \mathrm{p}$ and $13 \mathrm{p}$, translocations $(3 ; 15)$ and $(1 ; 6)$. He was treated with Revlimid and carfilzomib for seven cycles and switched to bortezomib and dexamethasone for two cycles. Patient achieved CR. He underwent a second stem cell transplant again in 2014, and was noted to be in clinical remission. Skeletal survey showed solitary lucent lesion in the left humerus without any other discrete abnormality. BM biopsy showed no increase in plasma cells $(<5 \%)$. No clonal plasma cells were identified by flow cytometry. Cytogenetic analysis showed a normal karyotype. In July 2015, patient presented with a nodular erythematous skin eruption involving face and chest (Fig. 1). The biopsy was positive for MM (Figs. 2, 3). Skeletal survey showed multiple lytic lesions in the axial and appendicular skeleton. Serum IgG was again elevated at $2,421 \mathrm{mg} / \mathrm{dL}$. Serum protein electrophoresis showed paraprotein peak. Serum immunofixation electrophoresis showed IgG kappa paraprotein peak. BM biopsy showed $75-80 \%$ monoclonal IgG kappa plasma cells. IgG kappa clonal plasma cell population was detected by flow cytometry. $\mathrm{Cy}-$

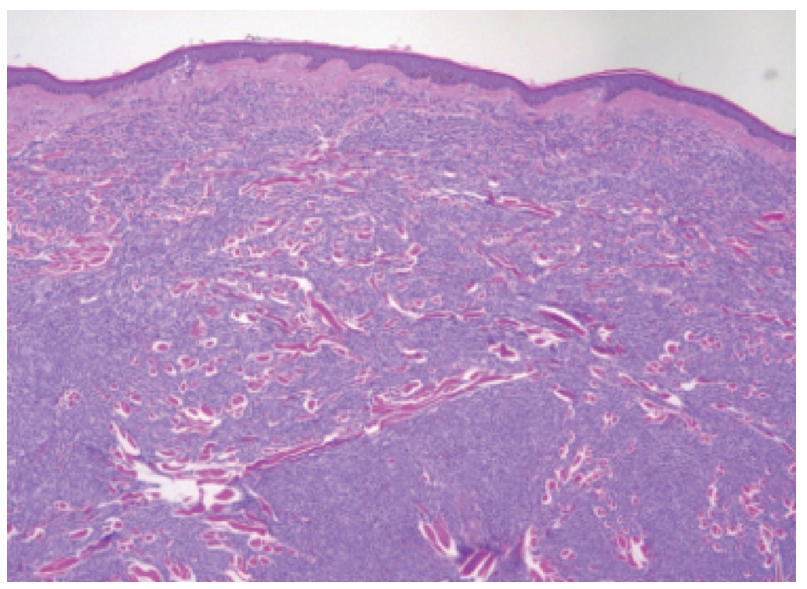

Figure 2. Biopsy showing dermal infiltration with plasma cells $(\times 50)$.

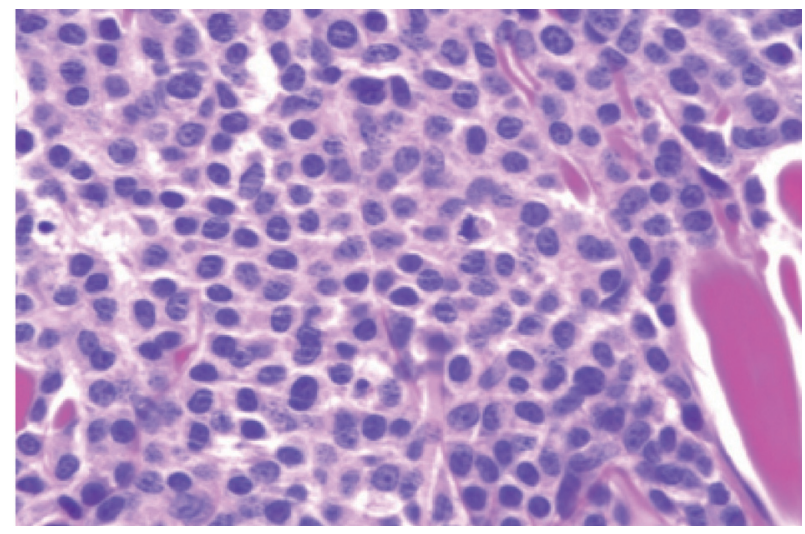

Figure 3. Biopsy showing atypical plasma cells $(\times 630)$.

togenetic analysis showed a complex abnormal karyotype including hypodiploidy, trisomy 1q21 and monosomy RB1 (13q14 deletion). He received Revlimid, cyclophosphamide and carfilzomib for seven cycles. Due to intolerance, the patient was switched to pomalidomide, panobinostat, and dexamethasone. Patient achieved a CR to treatment (Fig. 4) after 11 cycles.

In January 2018, patient developed new skin nodules. Repeat biopsy was positive for MM once again. Patient was treated with daratumumab for seven cycles with a CR to treatment with a partial resolution of his lesions and no new Mspike. Serum protein electrophoresis showed a small peak in the gamma region consistent with a paraprotein peak. Serum immunofixation electrophoresis showed a small IgG kappa paraprotein peak. Serum IgG level was normal. Patient was not a candidate for chimeric antigen receptor $\mathrm{T}$ cell (CAR-T cell) studies after he had a CR to daratumumab.

\section{Discussion}

MM is a malignancy of terminally differentiated plasma cells, and patients typically present with BM infiltration of clonal plasma cells and monoclonal protein in the serum and/or urine [4]. MM is the most frequent hematological cancer in the USA after non-Hodgkin lymphoma [5]. It is responsible for $1 \%$ of

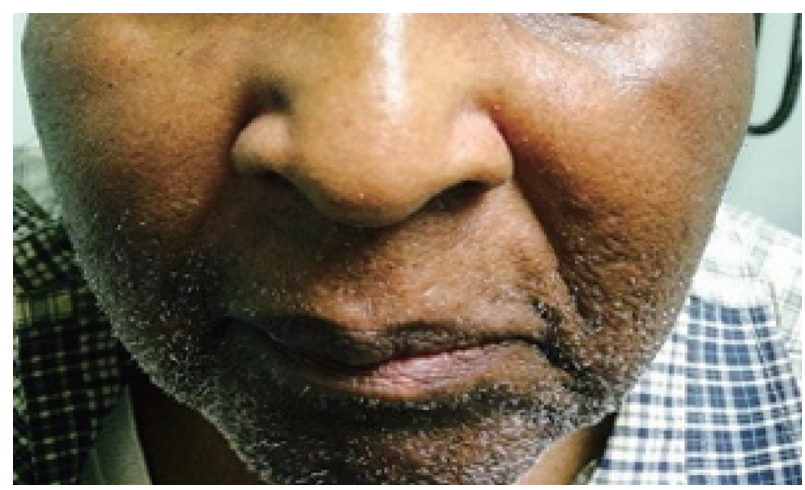

Figure 4. Resolution of cutaneous nodule after treatment with chemotherapeutic agents. 
all neoplastic diseases and $13 \%$ of all hematological malignancies [5]. The incidence is 7.1 and 4 cases per 100,000 for men and women, respectively. Mean age at diagnosis is 62 years for men and 61 years for women. It is more common in males than in females, and more common in African American patients than in white patients $[5,6]$. In a retrospective analysis of 1,027 patients diagnosed with MM, the signs and symptoms at presentation included anemia $(73 \%)$, bone pain $(58 \%)$, elevated creatinine $(19 \%)$, fatigue $(32 \%)$, hypercalcemia $(13 \%)$, and weight loss (24\%) [1]. Less common signs and symptoms include paresthesia $(5 \%)$, hepatomegaly $(4 \%)$, splenomegaly $(1 \%)$, lymphadenopathy $(1 \%)$ and fever $(0.7 \%)$. Indications for treatment are based on end-organ damage (hypercalcemia, renal impairment, anemia, bone lesions) and markers of active disease (serum free light chain ratio $\geq 100$, BM plasma cells $\geq$ $60 \%$, or $>1$ lesion found on magnetic resonance imaging) [7, 8]. Extramedullary MM represents $7-18 \%$ of newly diagnosed MM [9].

Skin manifestations in MM can be classified into three categories: 1) Primary extramedullary plasmacytoma; 2) $\mathrm{Cu}$ taneous plasma cell neoplasm in the setting of previously diagnosed MM; 3) Cutaneous plasma cell neoplasm from direct extension of osteolytic lesions in MM [10]. Primary extramedullary plasmacytoma is an extremely rare condition with an incidence of $2-4 \%$ of extramedullary cases. To establish this diagnosis, it requires absence of MM and plasmacytomas in other organs [11]. Cutaneous plasma cell neoplasm in the setting of previously diagnosed MM can involve skin as a result of a direct extension [11]. It is a very rare condition with incidence of $1.9 \%$ [12]. Skin plasmacytomas manifest as reddish, violaceous, non-tender dermal or subcutaneous nodules, and sometimes may also appear as diffuse erythematous rash [13]. Most common sites for cutaneous manifestations are chest (44\%), lower extremities (24\%), back and buttock (22\%), face and neck (20\%), and upper extremities (18\%) [2]. Forty percent of patients present in stage III, 32.5\% with stage II and 27.5\% with stage I [2]. Malysz et al (2016) identified 13 patients with cutaneous lesions of MM that consisted of pink, red, and violaceous papules, nodules, and/or plaques that varied in size [12]. The histopathology revealed atypical plasma cells with occasional plasmablastic features. Of clinical significance, most patients died of progressive disease within a few months after the development of cutaneous lesions despite aggressive management [12]. Behera et al (2016) described four cases who initially presented with common dermatoses such as leukocytoclastic vasculitis, pyoderma gangrenosum, and vesiculobullous disorders that were subsequently diagnosed to have MM [14]. It is reported that patients undergoing allogeneic transplantation with dose-reduced intensity conditioning have $37 \%$ of risk of extramedullary relapse [15]. However, patients with poor risk factors associated with higher probability of extramedullary relapse and/or clinically aggressive disease are offered allogeneic transplantation [3]. Extramedullary plasmacytoma can be triggered by a local extension of the tumor which is the most common way, a surgical procedure such as laparotomy, catheter insertion or hematogenous spread $[3,16]$. Extramedullary disease is usually associated with poor prognosis and shorter survival [17]. Usmani et al (2012) reported shorter overall survival of primary extramedullary myeloma (EM) patients when compared to MM patients (31\% vs. 59\% after 5 years) [18]. To the best of our knowledge, no prospective study of specific EM treatment has been published so far; thus, it is difficult to recommend a specific treatment strategy. However, Lonial et al reported EM should be treated as high-risk MM which includes high-level lactate dehydrogenase (LDH), high-risk cytogenetics or high-risk gene expression profiles [19]. For EM patients eligible for high-dose therapy, a triplet induction therapy followed by single or tandem peripheral blood stem cell transplant (PBSCT), a triplet consolidation therapy, and a maintenance treatment consisting of at least lenalidomide are suggested [19]. Radiotherapy should always be considered to improve local disease control and analgesia [20]. Thalidomide has no proven efficacy on extramedullary plasmacytoma [21]. On the other hand, there are several case reports on the efficacy of bortezomib on extramedullary plasmacytoma $[22,23]$. Our patient had received pomalidomide which is an analogue of thalidomide, an immunomodulatory agent, with several mechanisms of action. Pomalidomide is indicated for myeloma patients who have refractory or relapsed MM and who have received $\geq 2$ prior treatment regimens (including $\geq 2$ cycles of both lenalidomide and bortezomib). Like our patient, it is used in conjunction with dexamethasone [23]. To our knowledge there is no association between pomalidomide and successful treatment of extramedullary plasmacytoma. On the second relapse, our patient received daratumumab (CD38 antibody) and his cutaneous lesion completely resolved. According to our search, there is no study or case report on efficacy of daratumumab on cutaneous MM.

\section{Conclusions}

Extramedullary plasmacytoma involving the skin is rare and carries a poor prognosis. Hematogenous spread is the most common cause and likely mechanism of disease presentation and progression in our patient. Although less responsive to conventional treatment modalities and high mortality rate, the use of monoclonal antibodies (daratumumab, CD38 antibody) played pivotal role in better outcome of our patient. Focused research on use of monoclonal antibodies and their efficacy in such rare and fatal disease is needed on a larger scale.

\section{Acknowledgments}

We thank the Department of Hematology-Oncology and Pathology for their support and guidance. We thank Dr. Min Zheng, Pathology Department for providing us with biopsy images. We thank our librarian Chunwei Ma for providing us with other reported cases of cutaneous MM to compare to our patient.

\section{Financial Disclosure}

This project was not supported by any grant or funding agency. 


\section{Conflict of Interest}

None to declare.

\section{Informed Consent}

Written informed consent was obtained from the patient for publication of this case report and any accompanying images.

\section{Author Contributions}

Each author has individually been involved and participated in drafting the manuscript and revising it critically for important intellectual content, and have given final approval of the version to be published. MAH, KN and AM encouraged MM, AA and VG to learn about cutaneous MM and its management. All authors discussed the medical literature. MM presented the idea, AA, MM and VG wrote the manuscript with input from all authors.

\section{References}

1. Kyle RA, Gertz MA, Witzig TE, Lust JA, Lacy MQ, Dispenzieri A, Fonseca R, et al. Review of 1027 patients with newly diagnosed multiple myeloma. Mayo Clin Proc. 2003;78(1):21-33.

2. Jurczyszyn A, Olszewska-Szopa M, Hungria V, Crusoe E, Pika T, Delforge M, Leleu X, et al. Cutaneous involvement in multiple myeloma: a multi-institutional retrospective study of 53 patients. Leuk Lymphoma. 2016;57(9):20712076.

3. Blade J, Fernandez de Larrea C, Rosinol L, Cibeira MT, Jimenez R, Powles R. Soft-tissue plasmacytomas in multiple myeloma: incidence, mechanisms of extramedullary spread, and treatment approach. J Clin Oncol. 2011;29(28):3805-3812.

4. Kumar SK, Rajkumar V, Kyle RA, van Duin M, Sonneveld P, Mateos MV, Gay F, et al. Multiple myeloma. Nat Rev Dis Primers. 2017;3:17046.

5. Malpas JS, Bergsagel DE, Kyle RA, Anderson CS. Multiple Myeloma: biology and management. Oxford: Oxford University Press; 1998.

6. Cohen HJ, Crawford J, Rao MK, Pieper CF, Currie MS. Racial differences in the prevalence of monoclonal gammopathy in a community-based sample of the elderly. Am J Med. 1998;104(5):439-444.

7. Palumbo A, Anderson K. Multiple myeloma. N Engl J Med. 2011;364(11):1046-1060.

8. Rajkumar SV, Dimopoulos MA, Palumbo A, Blade J, Merlini G, Mateos MV, Kumar S, et al. International Myeloma Working Group updated criteria for the diagnosis of multiple myeloma. Lancet Oncol. 2014;15(12):e538-548.

9. Blade J, Lust JA, Kyle RA. Immunoglobulin D multiple myeloma: presenting features, response to ther- apy, and survival in a series of 53 cases. J Clin Oncol. 1994;12(11):2398-2404.

10. Carmel B, Delost GR, Stern J, Honda K. Cutaneous plasmacytoma: Metastasis of multiple myeloma and invasion of sternotomy scar. JAAD Case Rep. 2019;5(1):94-97.

11. Bhutani M, Shahid Z, Schnebelen A, Alapat D, Usmani SZ. Cutaneous manifestations of multiple myeloma and other plasma cell proliferative disorders. Semin Oncol. 2016;43(3):395-400.

12. Malysz J, Talamo G, Zhu J, Clarke LE, Bayerl MG, Ali L, Helm KF, et al. Cutaneous involvement in multiple myeloma (MM): A case series with clinicopathologic correlation. J Am Acad Dermatol. 2016;74(5):878-884.

13. Requena L, Kutzner H, Palmedo G, Calonje E, Requena C, Perez G, Pastor MA, et al. Cutaneous involvement in multiple myeloma: a clinicopathologic, immunohistochemical, and cytogenetic study of 8 cases. Arch Dermatol. 2003;139(4):475-486.

14. Behera B, Pattnaik M, Sahu B, Mohanty P, Jena S, Mohapatra L. Cutaneous Manifestations of Multiple Myeloma. Indian J Dermatol. 2016;61(6):668-671.

15. Perez-Simon JA, Sureda A, Fernandez-Aviles F, Sampol A, Cabrera JR, Caballero D, Martino R, et al. Reducedintensity conditioning allogeneic transplantation is associated with a high incidence of extramedullary relapses in multiple myeloma patients. Leukemia. 2006;20(3):542545.

16. De Larrea CF, Rosinol L, Cibeira MT, Rozman M, Rovira M, Blade J. Extensive soft-tissue involvement by plasmablastic myeloma arising from displaced humeral fractures. Eur J Haematol. 2010;85(5):448-451.

17. Wu P, Davies FE, Boyd K, Thomas K, Dines S, Saso RM, Potter MN, et al. The impact of extramedullary disease at presentation on the outcome of myeloma. Leuk Lymphoma. 2009;50(2):230-235.

18. Usmani SZ, Heuck C, Mitchell A, Szymonifka J, Nair B, Hoering A, Alsayed Y, et al. Extramedullary disease portends poor prognosis in multiple myeloma and is overrepresented in high-risk disease even in the era of novel agents. Haematologica. 2012;97(11):1761-1767.

19. Lonial S, Boise LH, Kaufman J. How I treat high-risk myeloma. Blood. 2015;126(13):1536-1543.

20. Touzeau C, Moreau P. How I treat extramedullary myeloma. Blood. 2016;127(8):971-976.

21. Patriarca F, Prosdocimo S, Tomadini V, Vasciaveo A, Bruno B, Fanin R. Efficacy of bortezomib therapy for extramedullary relapse of myeloma after autologous and non-myeloablative allogeneic transplantation. Haematologica. 2005;90(2):278-279.

22. Paubelle E, Coppo P, Garderet L, Azizi L, Bories D, Gorin NC, Fouillard L. Complete remission with bortezomib on plasmocytomas in an end-stage patient with refractory multiple myeloma who failed all other therapies including hematopoietic stem cell transplantation: possible enhancement of graft-vs-tumor effect. Leukemia. 2005;19(9):1702-1704.

23. Hoy SM. Pomalidomide: a review in relapsed and refractory multiple myeloma. Drugs. 2017;77(17):1897-1908. 